\title{
Commentary on papers to investigate the international assessment of mathematical knowledge for teaching
}

\author{
Ronald K. Hambleton
}

Accepted: 13 May 2012/Published online: 3 June 2012

(C) FIZ Karlsruhe 2012

The guest editors and contributors to this issue of ZDM on the topic of mathematical knowledge for teaching (MKT) are to be congratulated for the quality of their research, for their contributions to the effective teaching of mathematics and success of students, and for the interest and further research these five papers will undoubtedly generate. There are many aspects of the papers to like and one of them is that the papers describe teacher education work moving forward in five countries-Ghana, Iceland, Indonesia, Ireland and Korea. My prediction is that we will see both an expansion of this type of research in other countries, and we will see teacher education researchers in science, reading, social studies, and other subjects developing variations of the MKT instrument for their own use. I believe this collection of papers and the research already published by Deborah Ball, Sean Delaney, and their colleagues, is going to have an immense impact on teacher education research.

What made the papers especially interesting to me is that all of the papers addressed the important methodological problem of translating and adapting instruments from one language and culture to another. The papers are about relevant methodologies and their findings. Because of the influence of these papers not only on the substantive issues of teaching mathematics, but on the methodology of adapting instruments, I wish the papers had acknowledged or referenced the International Test Commission (ITC) Guidelines for Test Adaptation (http://www.itctestcom.org). These validated guidelines have been used in many countries and were developed by seven international

R. K. Hambleton ( $\square)$

School of Education, University of Massachusetts Amherst, 813 North Pleasant Street, Amherst, MA 01003, USA

e-mail: rkh@educ.umass.edu organizations of psychologists. They are frequently cited in the international testing literature, and because of their popularity, a second edition has been prepared and is about to be released. These ITC Guidelines would have provided a framework for each of the research teams (and research teams to come) and I think the methodologies used by the research teams would have been even better and more useful. Instruments like the MKT are increasingly being adapted to support an expansion of research and often to carry out cross-cultural research. As useful as I think the ITC Guidelines are, I think the work by Delaney (cf. 2008 and this issue) is an outstanding example too of test adaptation methodology. That the researchers in this issue took their inspiration from the Delaney et al. study is very important too.

Overall, I think the papers used excellent methodologies, and the adapted instruments are going to be valuable to researchers in these five counties as well as the United States for many years to come. At the same time, some methodological changes would have improved the use of translators and judgmental designs in this work. Translators need to know the languages and the cultures involved in the adaptation. It is surprising to note that these characteristics are not always respected, and the educational and psychological journals are littered with papers that did not follow these guidelines. Ype Poortinga, a well-known cross-cultural psychologist and past editor of the Journal of Cross Cultural Psychology, once noted that $80 \%$ of the cross-cultural research prior to 1990 should not have been published because of the poor quality of the translations and adaptations (see Hambleton, Merenda, \& Spielberger, 2005). It is desirable too that translators know something about the construct being investigated and instrument development. Without these skills they are not well equipped to carry out an effective translation and 
adaptation. I think these criteria may have been met, but the qualifications of translators were not made explicit in several of the papers.

The ITC Guidelines are also explicit about the desirability of "forward translation designs" and/or both "forward translation designs" and "backward translation designs" in the process. With the much more desirable forward translations design, translators focus their attention on the source and target language versions of the instrument and identify and subsequently revise problematic aspects of an adaptation. Often too, and usually, prior to the use of a forward translation design, a backward translation is used where the instrument is adapted from the source to the target language, and back translated to the source language and then the original source language and backtranslated version are compared, problems identified, and then appropriate revisions are made in the target language version of the instrument, if necessary. This preliminary design has a major problem in that there is not a focus on the target language version in the review. Fortunately, often problems are identified from the back translation design and any corresponding problems in the target language version of the instrument can be fixed prior to the more useful application of the forward adaptation design. But normally the forward translation design is more revealing of problems. Unfortunately, not all researchers follow up their back translation work with the use of a forward translation design. One common myth about translation and adaptation work is that a backward translations design is sufficient, when it is not.

Often these forward translation designs are best applied with at least four to six translators. At the same time, I do recognize the cost that can be associated with this process. On the other hand, if this step of translation, adaptation, and verification is not handled with great care, then the empirical analyses that follow will be reduced in value because of undetected flaws during the test adaptation process. But generally, I felt the adaptations were well done in the studies, but I would have preferred to see more documentation in the papers about what exactly was done. Perhaps limited journal space can be one problem for researchers, and if this is the case, researchers could place their full reports in a convenient place on the internet, and direct readers who want to know more about the methodologies to go there.

In general, from our experience, one of the biggest and most important flaws in the test adaptation validation process is the failure to carry out empirical analyses using scores obtained from the adapted instrument and comparing them in meaningful ways to scores and results obtained using the source language version of the instrument. Far too many projects end with the compilation of judgmental review evidence. One of the common myths about test adaptation is that a good translation is sufficient to validate an instrument for use in another language and cultural group. As is certainly clear from the five studies, all of them focused on empirical analyses, and a great amount of information was learned about the adaptation process from the empirical investigations. This, I think, is one of the important messages from the papers and that is confirmation about the need to carry out empirical studies to further investigate the quality and suitability of the instrument adaptation.

In all cases, the five research teams moved forward and compiled various types of validity evidence. As noted, in one of the papers, often these studies are not well funded, and so the available data with the target language version of the instrument is often limited. This can be problematic because it limits the kinds of analyses that are carried out and subsequently the amount of validity evidence that is available. Researchers must work even harder to compile substantial sample sizes for their empirical investigations.

In my opinion, these five papers are filled with important examples of empirical studies that were carried out to support the validity of the test adaptation process. Delaney (in this issue), for example, adopted methods from both Messick (1989) and Kane (2006), two of the most influential writers in the world on the topic of test validation, to provide a comprehensive approach to compiling empirical evidence to support the validity of scores from adapted instruments. I have seen these methodologies used to validate national and state assessment tests, and credentialing exams, but the application to adapted instruments is new to me, and highly appropriate. I was tremendously impressed, and hope that the example provided in Delaney's paper will be adopted by other researchers. I know that his test adaptation work will improve my own work in the coming years.

Also, the "argument-based" validation approach was adopted by Cole in her research, and the step by step application is a wonderful example of how researchers can go about compiling their validity evidence. Researchers are forced to state the claims that they are making about the scores from an adapted instrument, and then compile the relevant evidence to investigate the claims. The main methodological advancement in this study was the use of cognitive interviews to determine the understanding of teachers regarding the questions in the MKT as well as the mathematics. It is only recently that researchers in the test adaptation field have been using such methodologies, though cognitive interviews have been used successfully for years in validating scores from both educational and psychological tests and questionnaires. Again, I think both the Kane approach to building an argument about an instrument and its use, and then compiling evidence to support the argument as well as the use of cognitive 
interviews will highlight for others, the value of the approach in compiling evidence to support the use of an adapted instrument in a particular environment.

I was especially pleased too to see that Cole identified a number of method factors which are often difficult to spot statistically but when they go undetected, introduce a systematic error that makes individuals using the target language version of a test to score systematically lower or perhaps higher than individuals using a second language version of the test. I am always looking for examples of method biases to share with other researchers-Cole identified two. The first concerned a mismarking of the test answer sheet by some teachers because of some confusion between the labeling of the answer choices in the instrument itself and the labeling of choices on the answer sheet. This problem was identified during the cognitive interviewing. It might have been anticipated, but the problem was confirmed during the interviews. The second concerned the length of the instrument. A question arose about whether $150 \mathrm{~min}$ was too long to expect teachers to spend taking the instrument. In the American sample, for example, teachers had taken about $90 \mathrm{~min}$ to complete the instrument. Statistical methods can be quite helpful in spotting items showing potential biases or structural variations across language groups and cultures. Method factors are more difficult to spot because they are confounded with real group differences. Cognitive interviewing seems like a very promising way to spot systematic errors often called "method biases". Other biases might include a failure on the part of participants to understand the assessment format (e.g., multiple choice test questions), the directions themselves, the use of a rating scale, etc.

Very often in test adaptation validation work, researchers will investigate the factorial equivalence of a test across language versions. But somewhat larger sample sizes are needed than was available to most of the researchers in these five studies. A rough rule of thumb is that you would like to have a sample size that is at least ten times bigger than the number of items in the instrument. I don't know how realistic larger samples of respondents will be, or how available the funding may be to complete these studies, but establishing the factorial equivalence of an instrument across language groups is important validity evidence and fairly routine in practice.

Conducting what are called "differential item functioning (DIF)" studies are important too because items within an instrument can be spotted when candidates matched on total test score on the instrument show a pattern where candidates administered one language version perform differently than candidates perform on the other. It is not a problem that candidates in one language group might perform better than candidates in another on the total test score (often this comparison is of key importance in the research) because comparisons are made at the item level based on respondents matched on total test score. Valuable as these analyses are, they do require sample sizes that are somewhat larger than those used in these studies. Two hundred persons per language group is often a goal for these studies, though they can be carried out with fewer if (1) the performance of the two groups is not too far apart, and/or (2) the size of the DIF is substantial (Muñiz, Hambleton, \& Xing, 2001).

Instead of these factor analytic studies (often called today "structural equation modeling studies") or DIF studies, several of the researchers used other analyses with varying degrees of success. $\mathrm{Ng}$, for example, looked at a plot of point biserial correlations for items in the two language versions. The actual average correlation was not very high $(r=.37)$ but then point biserial correlations are not very stable with small samples, and they are influenced by the level of item difficulty and the homogeneity of the total test scores. I noted too that in the US sample that the point biserial correlations were quite high and similar, so I'm just not sure about the utility of the plot of point biserial correlations for the reasons given above. I can certainly imagine situations where the test adaptation was well done, and the plot of point biserials would not show the desired linear pattern of results indicating no problematic items.

$\mathrm{Ng}$, also, looked at the potentially more useful plot of item difficulties and the plot was helpful in spotting several problematic items. The correlation of the item difficulties across language and cultural groups may be useful to know, but more interesting is the use of the plot to spot outliers, and this was done. I am less enthusiastic about the comparison of test score reliabilities from the two language versions because the amount of test score variability in each group drives the value of reliability estimates. Well adapted instruments could exist and score reliabilities could be quite different in the two language groups, and the opposite is quite possible too-poorly adapted instruments could still lead to a similar test reliability estimate in each language group. In sum, I think plots of classical discrimination indices, as well as comparisons of test score reliabilities have some merit in the test adaptation validation process, but I think any results are preliminary, and have multiple possible interpretations, only one of which is flawed adaptations. On the other hand, the plot of item difficulty indices and watching for outliers in the scatterplot can be quite valuable I think.

Even the well-known comparison of rankings of item difficulties in the two language and cultural groups can be revealing in spotting items that are showing shifts in their rankings. These would be items worthy of additional investigation. They could be items showing flaws in their adaptation, or they could be items of considerable interest 
in looking for substantive explanations. A look through the history of methodologies for investigating problematic translations and adaptation would reveal the frequency with which the ranking analysis has been used.

The ITC Guidelines for Test Adaptation address the importance of documentation when an instrument is adapted from one language and culture to another. The paper by Kwon et al. is especially effective, I think, in this regard, and provides substantial details on the process of test adaptation. For example, the author provides a very useful table, in the framework of Delaney and his colleagues' work (Delaney et al., 2008), about areas in which changes to the language should be investigated, and later in the paper provided great detail on a number of changes made to test items. I think Kwon and colleagues are quite correct in providing this level of detail for readers. The details are interesting and informative.

Perhaps a final word about the use of item response theory (IRT) models in test adaptation research. Like Fauskanger et al., I too, like this approach, and I especially like to do plots of item difficulties to spot problematic items. At the same time, comparison of item statistics across language groups when each language and cultural group is on a separate IRT scale, is problematic, because each scale is unique to the sample of respondents that is used in scaling the data. Before comparisons are made, the scales need to be "linked" and this can be done as all items are potentially "anchor items," and the plot of b values, can be used to move the data from the scale for one language and cultural group to the scale for the second. Then, comparisons of both scores and items in the two language and cultural groups can be made on a common scale (for more details, see Hambleton, Merenda, \& Spielberger, 2005). Choice of IRT model should be made after determining that the chosen model fits the data. A comparison of the item characteristic curves for items in the two language and cultural groups reported on the same scale is an example of the DIF analysis I described earlier and can be very useful. But model fit needs to be established first, and the linking process (e.g., mean, sigma equating) needs to be carried out.

In sum, I think that this collection of papers investigating MKT presents some wonderful cross national results which are going to be well received by teacher education researchers around world, and it was a pleasure to have the chance to read the papers. I think too that the papers are going to stimulate more instrument adaptation initiatives, and I hope readers are impressed and will generally follow the ambitious efforts to validate the MKT and instruments like it. In general, the researcher should carry out both empirical as well as judgmental reviews, and try to use large samples and some of the more suitable statistical procedures. For more information on the ITC Guidelines for Test Adaptation, and applications of emerging methodologies, readers are encouraged to read the chapters in Hambleton, Merenda, and Spielberger (2005) in addition to the excellent work reflected in the five papers and the outstanding work by Delaney and his colleagues (2008).

\section{References}

Delaney, S. (2008). Adapting and using US measures to study Irish teachers' mathematical knowledge for teaching. Unpublished doctoral dissertation, University of Michigan, Ann Arbor, MI.

Delaney, S., Ball, D. L., Hill, H. C., Schilling, S. G., \& Zopf, D. (2008). Mathematical knowledge for teaching: adapting U.S. measures for use in Ireland. Journal of Mathematics Teacher Education, 11, 171-197.

Hambleton, R. K., Merenda, P., \& Spielberger, C. (Eds.). (2005). Adapting educational and psychological tests for cross-cultural assessment. Mahwah, NJ: Lawrence Erlbaum.

Kane, M. (2006). Validation. In R. L. Brennan (Ed.), Educational measurement (4th ed, pp. 17-64). Westport, CT: American Council on Education and Praeger Publishers.

Messick, S. (1989). Meaning and values in test validation: the science and ethics in assessment. Educational Researcher, 18(2), 5-11.

Muñiz, J., Hambleton, R. K., \& Xing, D. (2001). Small sample studies to detect flaws in item translations. International Journal of Testing, 1(2), 115-135. 